OPEN ACCESS

Edited by:

Massimo Breccia

Sapienza University of Rome, Italy

Reviewed by:

Fausto Castagnetti,

University of Bologna, Italy

Valentin Garcia-Gutierrez,

Ramón y Cajal University Hospital,

Spain

*Correspondence:

Honghu Zhu

zhuhhdoc@163.com

Jie Jin

jiej0503@zju.edu.cn

Liangshun You

youliangshun@zju.edu.cn

${ }^{\dagger}$ These authors share first authorship

Specialty section:

This article was submitted to Hematologic Malignancies,

a section of the journal

Frontiers in Oncology

Received: 07 July 2020 Accepted: 10 November 2020 Published: 15 December 2020

Citation:

Lin Q, Mao L, Shao L, Zhu L, Han Q, Zhu H, Jin J and You L (2020) Global, Regional, and National Burden of Chronic Myeloid Leukemia, 19902017: A Systematic Analysis for the Global Burden of Disease Study 2017.

Front. Oncol. 10:580759. doi: 10.3389/fonc.2020.580759

\section{Global, Regional, and National Burden of Chronic Myeloid Leukemia, 1990-2017: A Systematic Analysis for the Global Burden of Disease Study 2017}

\author{
Qingqing Lin ${ }^{1,2 \dagger}$, Liping Mao ${ }^{1,2 \dagger}$, Li Shao ${ }^{1,2 \dagger}$, Li Zhu ${ }^{1,2}$, Qingmei Han ${ }^{1,3}$, Honghu Zhu ${ }^{1,2 *}$, \\ Jie Jin $^{1,2 *}$ and Liangshun You ${ }^{1,2 *}$ \\ ${ }^{1}$ Department of Hematology, The First Affiliated Hospital, College of Medicine, Zhejiang University, Hangzhou, China, \\ ${ }^{2}$ Malignant Lymphoma Diagnosis and Therapy Center, The First Affiliated Hospital, College of Medicine, Zhejiang University, \\ Hangzhou, China, ${ }^{3}$ Department of Pathology, Zhejiang University, Hangzhou, China
}

Background: With the advent of tyrosine kinase inhibitors (TKIs), the prognosis of chronic myeloid leukemia (CML) seems to have dramatically improved over the last two decades. Accurate information of the global burden of $\mathrm{CML}$ is critical for direct health policy and healthcare resource allocation in the era of high-cost TKI therapy.

Objective: This study aimed to evaluate the health burden of CML at global, regional, and national levels from 1990 to 2017.

Methods: We collected data of CML between 1990 and 2017 from the Global Burden of Disease (GBD) study 2017 including, annual incidence, disease-related mortality, and disability-adjusted life-years (DALY), and the corresponding age-standardized rates (ASRs). To summarize the results, countries were categorized by sociodemographic index (SDI) quintiles and $21 \mathrm{GBD}$ regions.

Results: In 2017, an estimated 34,179 [95\% Uncertainty Interval (UI), 31,516-36,714) incident cases of CML were recorded, and 24,054 (95\%UI, 22,233-26,072) CML-related deaths were reported worldwide. Both, the age-standardized incidence rate (ASIR) and age-standardized death rate (ASDR) steadily decreased from 1990 to 2017, with estimated annual percentage changes (EAPCs) of -2.39 (95\%Ul, $-8.13-3.71)$ and -2.74 (95\%UI, $-9.31-4.31)$, respectively. The global incidence and mortality of CML in males were higher than that in females. The ASRs varied substantially across regions, with 
the highest burden in Andean Latin America, Central Sub-Saharan Africa, and Southeast Asia. Besides, the ASRs decreased most obviously in the high-SDI regions compared to non-high-SDI regions. Moreover, the lower the SDI, the higher was the proportion of deaths in the younger age groups.

Conclusion: Despite the decreasing trends of ASRs of CML from 1990 to 2017, the health-related burden of CML remains a challenge for the low-SDI regions. These findings highlight that appropriate strategies should be adopted in low-SDI countries to reduce the ASRs of CML.

Keywords: chronic myeloid leukemia, epidemiology, incidence, death, disability-adjusted life-years

\section{INTRODUCTION}

Chronic myeloid leukemia (CML) is a clonal hematopoietic stem cell disorder and accounts for approximately $30 \%$ of the incidence of adult leukemias (1). Life expectancy for patients with CML has substantially improved since the advent of tyrosine kinase inhibitors (TKIs) at the turn of the century (2). Of note, the prognosis of CML has changed from a fatal disease to a disorder that is compatible with a normal lifespan (3). As a result, the prevalence of CML has increased dramatically, and may reach a plateau in 2050s (4). However, the high-cost TKI treatment combined with the rising prevalence of CML has led to a high global burden of CML treatment. Therefore, comparable epidemiological statistics such as age-standardized rates (ASRs) are important metrics to assess the global burden of CML in different countries at various levels of economic development, which may show potentially distinct patterns that can direct health policy and health care resource allocation in the era of high-cost TKI therapy.

At present, available data shows that the CML incidence distribution varies with age, gender, and regions $(1,5)$. The annual incidence of CML varies from $0.4 / 100,000$ persons to $1.75 / 100,000$ persons in different countries $(6-8)$. The incidence of CML increases with age and is rare in children less than 14 years of age ( $0.7 /$ million children/year) (9). Besides, CML is more common in males than in females with the male/female ratio of $1.2-1.7(6,10,11)$. However, to the best of our knowledge, no studies have characterized the distribution and burden of CML worldwide.

The Global Burden of Disease (GBD) study from the Global Health Data Exchange (GHDx) assessed the burden of 354 diseases and injuries in $21 \mathrm{GBD}$ regions and 194 countries worldwide. In this study, we collected data of CML between 1990 and 2017 from the GBD 2017 study, including incidence, disease-related mortality, disability adjusted life-years (DALYs), and the corresponding ASRs across gender, age, socio-demographic index (SDI), region, and country. Next, estimated annual percentage changes (EAPCs) were calculated to quantify the trends of ASRs. This was aimed to assess the accurate assessment of the distribution, burden, and trends of CML in different regions and countries that may provide directions to allocate limited resources and formulate more rational policies.

\section{METHODS}

\section{Data Source and Collection}

All GBD 2017 data resources were available online from the GHDx query tool (http://ghdx.healthdata.org/gbd-results-tool). Regarding CML, we collected the information on incidence rate, death rate, and DALYs that was sorted by age, gender, and location from 1990 to 2017. The sociodemographic index (SDI), which was measured by the geometric average of total income per capita, educational attainment, and fertility, was used to divide the 194 countries into high-SDI, high-middle-SDI, middle-SDI, low-middle-SDI, and low-SDI countries. Based on a geographical hierarchy, GBD 2017 grouped the 194 countries into 21 regions. Therefore, the differences of CML burden and trends across these countries and regions were analyzed (12-14).

\section{Statistical Analysis}

The annual age-standardized incidence rate (ASIR), age standardized death rate (ASDR), age-standardized DALY rate, and the corresponding estimated annual percentage changes (EAPCs) were calculated to assess the burden of CML prevalence. To assess the disease burden, the index of DALYs, which was estimated by addition of the YLDs and the years of life lost (YLLs), was applied. To assess the effect of the development status on the CML burden, we analyzed the association between SDI and prevalence indexes. The 95\% Uncertainty Interval (UI) for each quantity was estimated in the analyses. The EAPCs were used to evaluate the trends of ASRs, which were calculated using a regression model: $\mathrm{y}=\alpha+\beta \mathrm{x}+\epsilon$, where $\mathrm{y}=\ln$ (rate), $\mathrm{x}=$ calendar year, and $\epsilon=$ error term. EAPC $=100 \times\left(\exp ^{(\beta)}-1\right)$ and its $95 \%$ UI was obtained from the regression model $(13,15)$. If the EAPC and lower limit of UI were negative values, the incidence rate was considered to have a descending trend; in contrast, if the EAPC and upper limit of UI were positive, the incidence rate was considered to have an ascending trend. All calculations and analysis were performed using the R software (version 3.6.3). All tests were twotailed, and a $P$-value of $<0.05$ was considered statistically significant.

\section{RESULTS}

\section{Temporal Trends on Incidence of CML}

Globally, from 1990 to 2017, the number of annual incident cases of CML was stable, and there were 31,752 (95\%UI, 
29,590-34,066) cases in 1990 and 34,179 (95\%UI, 31,51636,714 ) cases in 2017 (Table 1), with a total increase of $7.64 \%$. Contrarily, the ASIR decreased steadily, from 0.75 per 100,000 persons (95\%UI, $0.71-0.8$ ) in 1990 to 0.43 per 100,000 persons (95\%UI, 0.4-0.46) in 2017. The EAPC $(-2.39,95 \% U I$, -8.13-3.71) was negative, pointing to an obviously descending trend in the global ASIR over the past 28 years, especially since 1997 (Table 1, Figure 1A, Supplementary Table S1). CML occurred almost exclusively after the age of 20 years, and the incidence increased with age, both in males and females (Table 1, Figures 2A, 3A, C, 4A, D, Supplementary Tables S1, S4, S5, S7, S9, S10). However, the Global ASIR in males was higher than that in females over the past 28 years [0.52 (0.47-0.57) vs. 0.36 (0.31-0.41), respectively, in 2017] (Table 1, Supplementary Table S1). The ratio of the incidence between males and females showed a peak in the age group of 75-80 years, and dropped sharply thereafter (Figure 2A, Supplementary Table S4).

The SDI-based regional analysis showed that the CML incidence was the highest in the high-SDI regions in 1990 and decreased steadily every year, especially after 1997. As shown in
Table 1, the incident cases of CML in high-SDI regions were 16,342 (95\%UI, 15,715-16951) in 1990, while they were 10,640 (95\%UI, 10,095-11,218) in 2017, which were consistent with the trend of ASIR. Conversely, in the non-high-SDI regions, CML incident cases increased, whereas ASIR decreased. In addition, we also found a trend that the higher the SDI, the higher were the male/female ratio of incidence rates (Figure 2, Supplementary Table S4). It was remarkable that the proportion of younger ( $<60$-years-old) incident cases in the high-SDI region decreased each year from 1990 to 2017, which was lower than that in nonhigh-SDI regions in 2017 (Figure 3, Supplementary Table S5, S7).

While analyzing data for the 21 GBD regions, ASIR showed an upward trend in 3 GBD regions: Andean Latin America, Central Sub-Saharan Africa, and Southeast Asia, and a downward trend in the other 18 GBD regions. A significant decline of EAPC was observed in eight regions: Australasia, Central Europe, High-income Asia Pacific, High-income North America, Southern Latin America, Tropical Latin America, and Western Europe. Interestingly, compared with other regions, the proportion of younger patients $(<60$-years-

TABLE 1 | Incident cases and ASIR in 1990 and 2017 and its change trends from 1990 to 2017.

\begin{tabular}{|c|c|c|c|c|c|}
\hline & \multicolumn{2}{|c|}{1990} & \multicolumn{2}{|c|}{2017} & \multirow{2}{*}{$\begin{array}{c}\text { 1990-2017 } \\
\text { EAPC } \\
\text { No. }(95 \% \mathrm{Cl})\end{array}$} \\
\hline & $\begin{array}{l}\text { Incident cases } \\
\text { No. }{ }^{*} 10^{2}(95 \% \text { UI) }\end{array}$ & $\begin{array}{c}\text { ASIR } \\
\text { Per } 100,000 \\
\text { No. }(95 \% \text { UI) }\end{array}$ & $\begin{array}{l}\text { Incident cases } \\
\text { No. }{ }^{*} 10^{2}(95 \% \text { UI) }\end{array}$ & $\begin{array}{c}\text { ASIR } \\
\text { Per } 100,000 \\
\text { No. }(95 \% \text { UI) }\end{array}$ & \\
\hline Overall & 317.52 (295.9-340.66) & $0.75(0.71-0.8)$ & 341.79 (315.16-367.14) & $0.43(0.4-0.46)$ & $-2.39(-8.13-3.71)$ \\
\hline \multicolumn{6}{|l|}{ Sex } \\
\hline Female & $151.96(135.82-171.91)$ & $0.67(0.61-0.75)$ & 150.33 (130.96-170.43) & $0.36(0.31-0.41)$ & $-2.69(-8.84-3.87)$ \\
\hline Male & 165.57 (153.46-177.23) & $0.86(0.8-0.92)$ & 191.45 (173.2-208.22) & $0.52(0.47-0.57)$ & $-2.16(-7.47-3.45)$ \\
\hline \multicolumn{6}{|l|}{ Socio-demographic index } \\
\hline High SDI & 163.42 (157.15-169.51) & $1.34(1.29-1.38)$ & $106.4(100.95-112.18)$ & $0.53(0.51-0.56)$ & $-3.96(-8.61-0.93)$ \\
\hline High-middle SDI & $49.58(43.76-55.26)$ & $0.49(0.43-0.54)$ & 57.35 (52.42-62.39) & $0.33(0.3-0.36)$ & $-1.72(-8.44-5.46)$ \\
\hline Middle SDI & $38.31(34.17-44.49)$ & $0.33(0.3-0.39)$ & $67.71(58.04-72.69)$ & $0.30(0.26-0.32)$ & $-0.55(-8.25-7.80)$ \\
\hline Low-middle SDI & $32.8(28.42-41.59)$ & $0.48(0.42-0.61)$ & 56.67 (49.19-65.98) & $0.43(0.37-0.5)$ & $-0.47(-6.90-6.40)$ \\
\hline Low SDI & $32.8(25.9-41.69)$ & $0.81(0.67-1.01)$ & 52.85 (44.44-59.95) & $0.65(0.55-0.74)$ & $-0.92(-6.10-4.53)$ \\
\hline \multicolumn{6}{|l|}{ Region } \\
\hline Andean Latin America & $0.87(0.73-1)$ & $0.34(0.28-0.38)$ & $2.08(1.69-2.39)$ & $0.36(0.29-0.42)$ & $0.45(-6.92-8.42)$ \\
\hline Australasia & $3.87(3.36-4.44)$ & $1.64(1.43-1.87)$ & $3.58(2.95-4.32)$ & $0.83(0.68-1)$ & $-3.24(-7.18-0.86)$ \\
\hline Caribbean & $2.64(2.41-2.91)$ & $0.93(0.85-1.02)$ & $3.07(2.73-3.48)$ & $0.61(0.54-0.69)$ & $-1.74(-6.87-3.69)$ \\
\hline Central Asia & $2.52(2.16-2.88)$ & $0.45(0.39-0.51)$ & $2.74(2.41-3.07)$ & $0.32(0.28-0.36)$ & $-1.29(-8.10-6.02)$ \\
\hline Central Europe & $11.11(10.48-11.77)$ & $0.77(0.73-0.82)$ & $6.82(6.42-7.27)$ & $0.37(0.35-0.4)$ & $-2.74(-8.41-3.28)$ \\
\hline Central Latin America & $6.98(6.69-7.4)$ & $0.66(0.64-0.7)$ & $11.96(11.22-12.85)$ & $0.49(0.46-0.53)$ & $-1.42(-7.05-4.55)$ \\
\hline Central Sub-Saharan Africa & $1.49(0.99-1.92)$ & $0.51(0.35-0.64)$ & $3.61(2.64-4.51)$ & $0.57(0.42-0.72)$ & $0.58(-5.43-6.97)$ \\
\hline East Asia & $22.64(17.43-28.02)$ & $0.19(0.16-0.24)$ & $32.71(27.97-37.64)$ & $0.17(0.15-0.19)$ & $-0.99(-11.04-10.19)$ \\
\hline Eastern Europe & $18.01(15.98-20.72)$ & $0.66(0.59-0.75)$ & $18.51(16.46-20.67)$ & $0.61(0.54-0.69)$ & $-0.54(-5.89-5.11)$ \\
\hline Eastern Sub-Saharan Africa & $11.1(8.15-15.09)$ & $1.11(0.86-1.49)$ & 16.39 (12.56-19.89) & $0.81(0.63-0.98)$ & $-1.41(-5.88-3.29)$ \\
\hline High-income Asia Pacific & $17.08(15.68-18.57)$ & $0.87(0.79-0.94)$ & $12.81(11.33-14.46)$ & $0.39(0.34-0.44)$ & $-3.12(-8.69-2.79)$ \\
\hline High-income North America & 34.1 (32.98-35.37) & $1.02(0.98-1.06)$ & $20.55(19.44-21.81)$ & $0.4(0.37-0.43)$ & $-4.30(-9.68-1.39)$ \\
\hline North Africa and Middle East & $11.85(8.69-14.29)$ & $0.57(0.42-0.69)$ & $18.32(15.18-21.5)$ & $0.38(0.32-0.44)$ & $-1.58(-7.91-5.19)$ \\
\hline Oceania & $0.33(0.25-0.44)$ & $0.78(0.6-1)$ & $0.55(0.41-0.78)$ & $0.59(0.45-0.79)$ & $-1.06(-6.43-4.61)$ \\
\hline South Asia & 44.97 (38.05-55.49) & $0.64(0.55-0.79)$ & $80.44(69.27-91.61)$ & $0.56(0.48-0.63)$ & $-0.58(-6.27-5.46)$ \\
\hline Southeast Asia & $12.41(10.18-17.69)$ & $0.39(0.33-0.57)$ & $25.17(19.85-29.4)$ & $0.4(0.31-0.46)$ & $0.046(-6.90-7.51)$ \\
\hline Southern Latin America & $3.77(3.47-4.09)$ & $0.8(0.73-0.87)$ & $3.0(2.69-3.35)$ & $0.38(0.34-0.43)$ & $-3.12(-8.72-2.84)$ \\
\hline Southern Sub-Saharan Africa & $0.33(0.25-0.42)$ & $0.1(0.07-0.12)$ & $0.48(0.36-0.6)$ & $0.08(0.06-0.09)$ & $-0.69(-14.17-14.90)$ \\
\hline Tropical Latin America & $7.2(6.88-7.55)$ & $0.65(0.62-0.68)$ & $7.65(7.26-8.15)$ & $0.33(0.31-0.35)$ & $-2.76(-8.85-3.75)$ \\
\hline Western Europe & 99.5 (94.04-104.66) & $1.78(1.69-1.87)$ & $61.62(56.85-66.77)$ & $0.69(0.64-0.75)$ & $-4.06(-8.11-0.170)$ \\
\hline Western Sub-Saharan Africa & $4.74(3.75-5.92)$ & $0.43(0.34-0.54)$ & $9.72(7.87-12.33)$ & $0.42(0.33-0.52)$ & $-0.13(-6.91-7.14)$ \\
\hline
\end{tabular}

ASIR, age-standardized incidence rate; EAPC, estimated annual percentage change. 


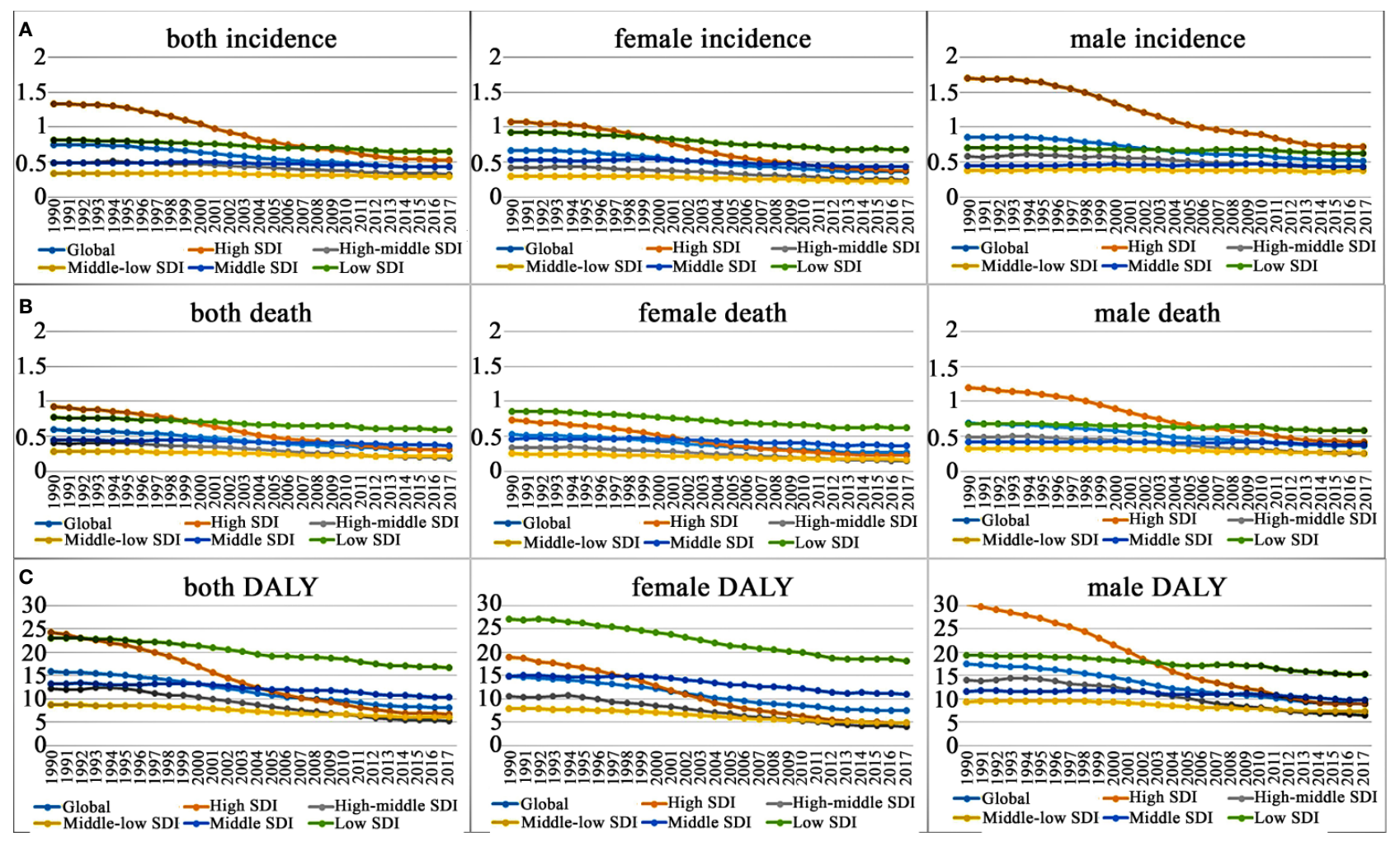

FIGURE 1 | Change trends in age-standardized incidence, death and DALY rate by gender in different SDI quintiles from 1990 to 2017. (A) Age-standardized incidence rate; (B) age-standardized death rate; (C) age-standardized DALY rate. SDI, socio-demographic index.

old) in these eight regions decreased most significantly. The ASIR in 2017 and trends of ASIR over 28 years in 194 countries are presented in Figure 5A, of which, six countries with the ASIR more than one in 2017 were: Ethiopia, Brunei, Honduras, Seychelles, Denmark, and Costa Rica, in descending order. The five countries with the highest EAPC were Jamaica, El Salvador, Ecuador, Philippines, and Zimbabwe, while the five countries with the lowest EAPC were Germany, UK, Hungary, Israel, and Puerto Rico (details are available in Supplementary Table S11).

\section{Trends on Deaths Attributable to CML}

The deaths attributable to CML were stable over the past 28 years with 24,198 (95\%UI, 22,632-26,108) deaths in 1990 and 24,054 (95\%UI, 22,233-26,072) deaths in 2017, while the ASDR decreased significantly with an EAPC -2.74 (95\%UI, -9.314.31 ), dropping from $0.59 / 100,000$ persons (95\%UI, $0.56-0.63$ ) to $0.31 / 100,000$ persons (95\%UI, 0.28-0.33) between 1990 and 2017 (Table 2). Consistent with the changes of ASIR, the ASDR increased with age in both sexes. However, ASDR in males was higher than that in females over the past 28 years (Figure 1B, Supplementary Table S2).

The SDI-based analysis revealed that the ASDR in highand high-middle-SDI regions decreased obviously each year, especially in the male subjects (Figure 1B, Supplementary Table S2). However, no significant changes were observed in the low- and middle-low-SDI regions. It was noteworthy that the low-SDI regions had the highest ASDR 0.6/100,000 persons (95\%UI, 0.5-0.68) (Table 2, Figure 1B, Supplementary Table S2) in 2017. Moreover, the lower the SDI, the higher was the proportion of deaths in younger groups (<60-years-old) (Figures 3B, D, Supplementary Table S6, S8).

The analysis at the level of GBD regions revealed that the ASDR in all regions declined over past the 28 years, except for Central Sub-Saharan Africa (0.55, 95\%UI, -5.65-7.15). Interestingly, the regions with the most obvious decrease of ASDR were the same as the regions with the most obvious decline of ASIR. Similarly, the top three countries in terms of ASDR were Ethiopia, Brunei, and Honduras, which also had the highest ASIR (Figure 6A, Supplementary Table S12). From 1990 to 2017, the ASDR declined in 168 countries (Figure 6B), of which nine countries with EAPC lower than -5 were Japan, Puerto Rico, UK, Hungary, Finland, Singapore, USA, Canada, and Bahrain. While, the seven countries with the highest EAPC (>1) were Jamaica, El Salvador, Zimbabwe, Ecuador, Lesotho, Philippines, and Georgia (details are available in Supplementary Table S12). 


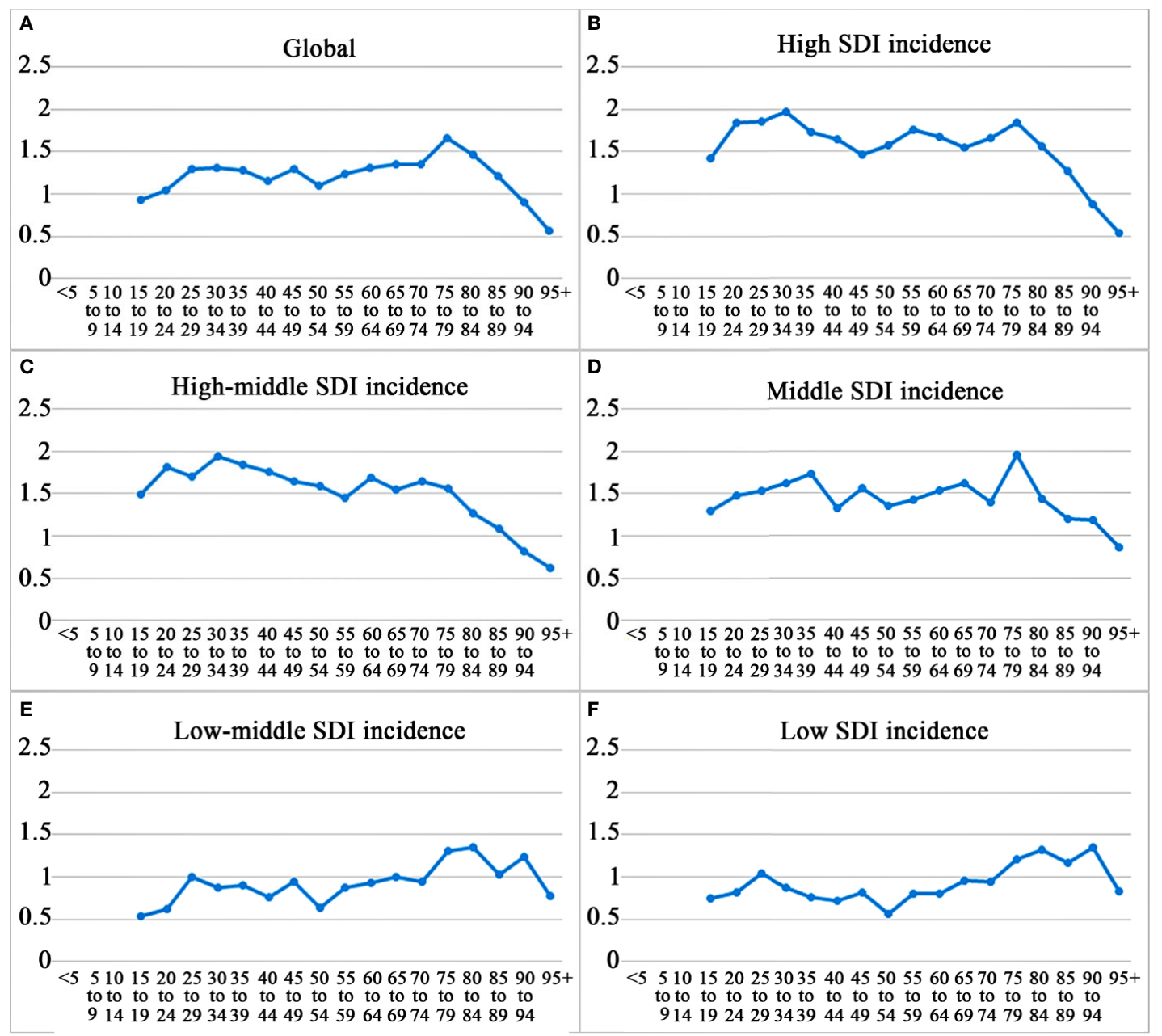

FIGURE 2 | The ratio of male to female by age groups in different SDI quintiles in 2017. (A) Global; (B) high SDI; (C) high-middle SDI; (D) middle SDI; (E) low-middle SDI; (F) low SDI.

\section{Trends on DALYs Attributable to CML}

The DALY attributable to CML decreased from 734,923 (95\%UI, 666,259 to 806,312 ) in 1990 to 654,983 (95\%UI, 594,726 to $712,947)$ in 2017. Simultaneously, the age-standardized DALY rate had fell significantly from 15.96 per 100,000 persons (95\% UI, $14.60-17.44$ ) to 8.17 per 100,000 persons (95\%UI, 7.42-8.88) with an EAPC of -2.82 (95\%UI, from -4.12 to -1.49$)$. The Global DALY in males was higher than that in females over the past 28 years, [9 (95\%UI, 8.01-9.9) vs. 7.48 (95\%UI, 6.27-8.68), respectively, in 2017] (Table 3, Figure 1C, Supplementary Table S3). ASDR increased with age in both sexes and achieved the peak in 80-84-years-old age group (Figures 4C, F, Supplementary Tables S3, S9, S10).

The age-standardized DALY rates declined in each SDI region over the past 28 years, especially since 1997 . However, the decline was more pronounced in high- and high-middleSDI regions (Table 3, Figure 1C, Supplementary Table S3). There was wide geographic variation in the age-standardized
DALY rate of CML. Regions with an increase were only observed in Central Sub Saharan Africa. Ethiopia, one country of Central Sub Saharan Africa, had the largest agestandardized DALY over the past 28 years and that of 54.13 per 100,000 persons (95\%UI, 38.02-69.90) in 2017 (Table 3, Supplementary Table S13). The top three countries of agestandardized DALY were Ethiopia, Brunei, and Honduras, which also had the highest ASIR and ASDR The seven countries with the highest EAPC $(>1)$ of age-standardized DALY were the same which also had the highest ASDR (Figures 7A, B, Supplementary Table S3).

\section{DISCUSSION}

Our analysis based on the GBD study 2017 presented the latest global patterns and long-term trends of the incidence, mortality, and DALYs attributable to CML. Overall, the cases 
A

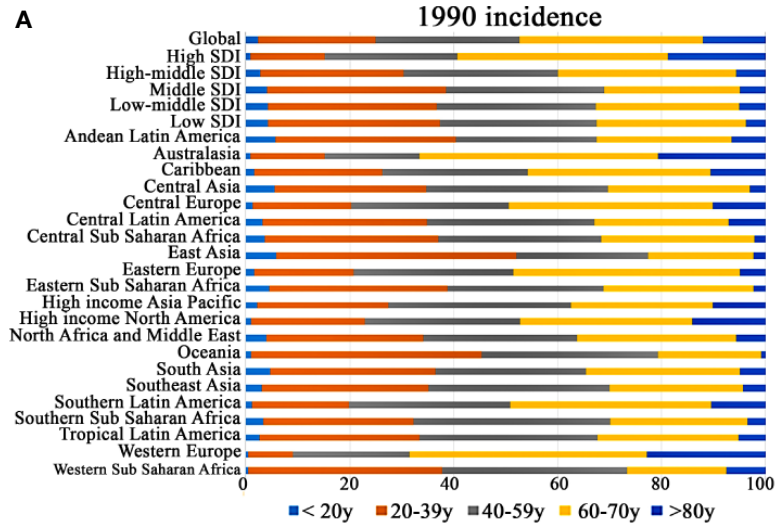

C

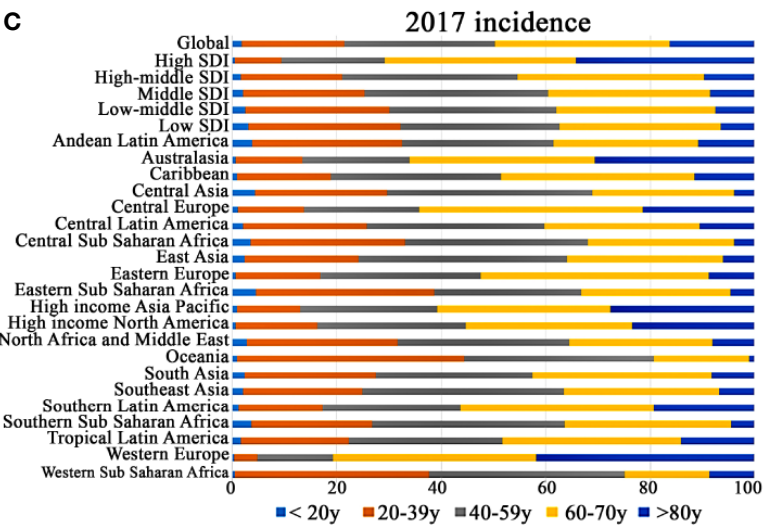

B

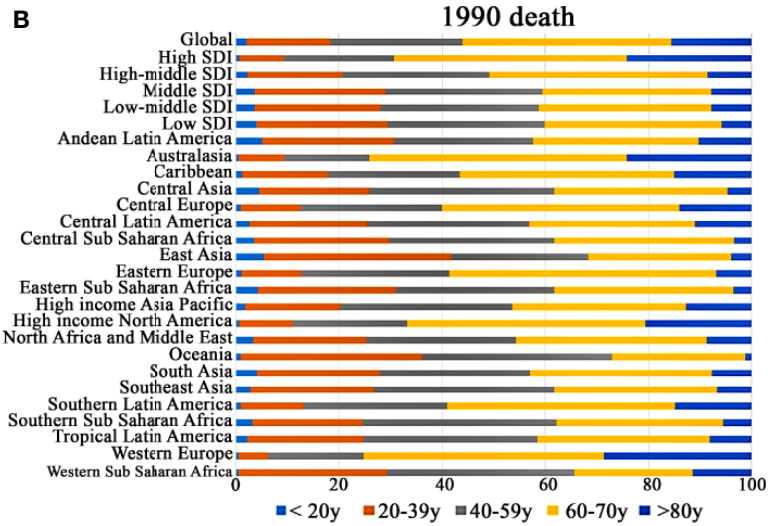

D

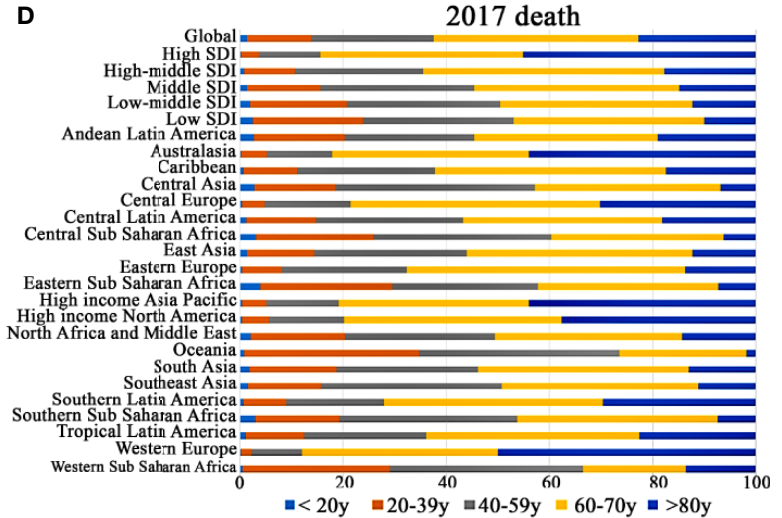

FIGURE 3 | Proportion of age groups on incidence and death by region in 1990 and 2017. (A) incidence in 1990; (B) incidence in 2017; (C) death in 1990; (D) death in 2017
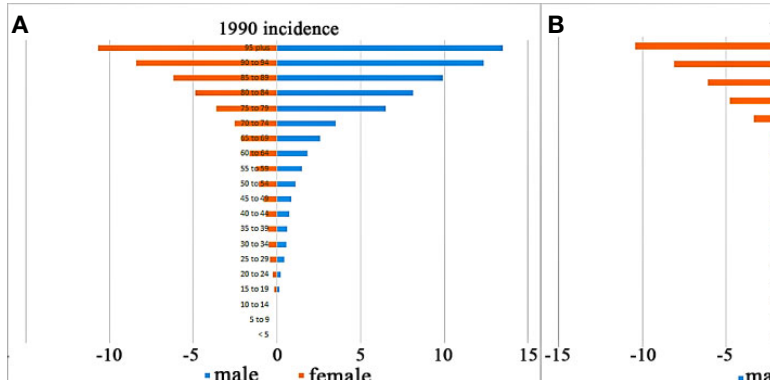

1990 death
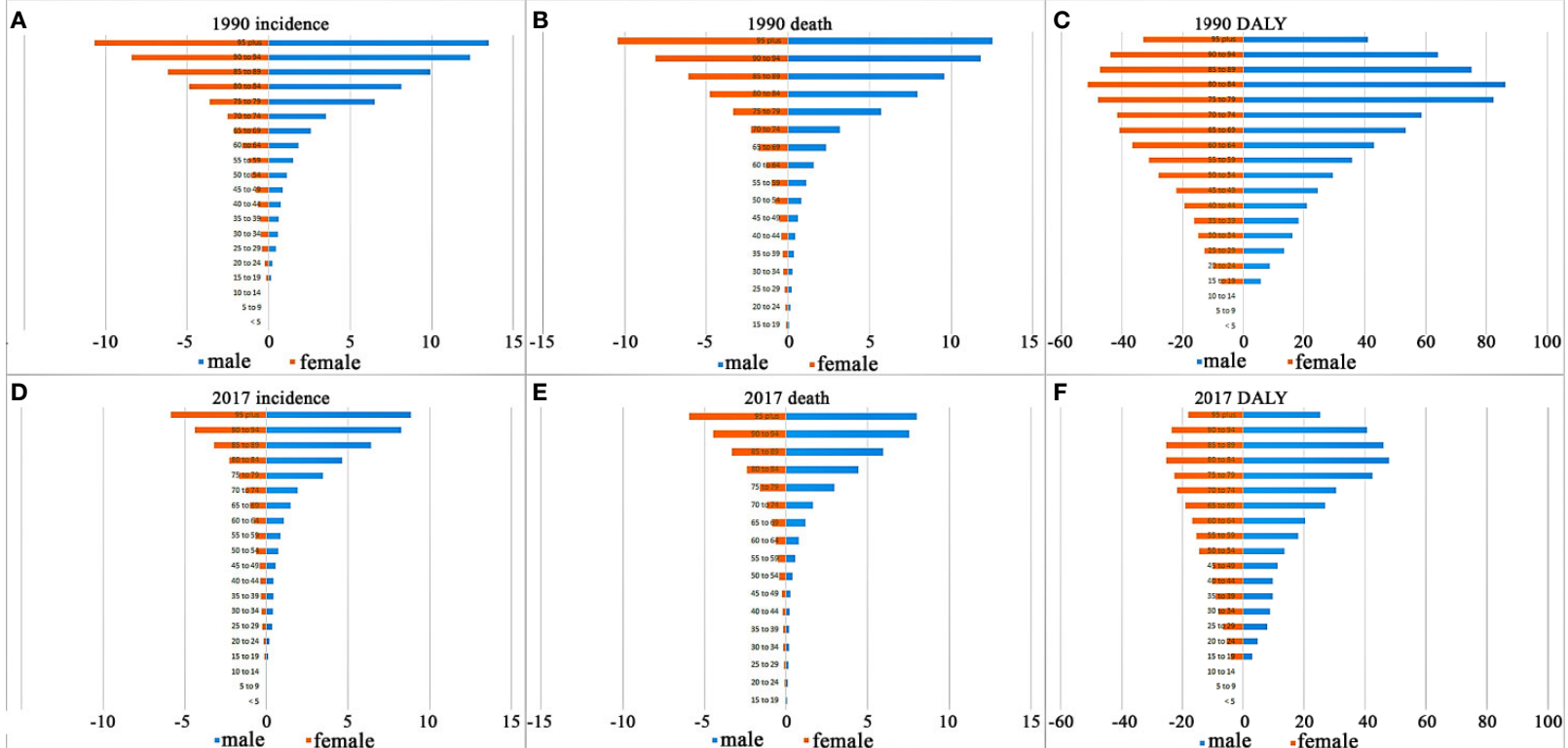

$\mathbf{F}$

2017 DALY

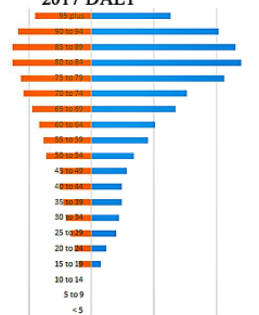

$-20 \stackrel{0}{20}{ }_{\text {male }}^{20}{ }^{40}$

$80 \quad 100$

FIGURE 4 | The incidence, death, and DALY rates by gender and age groups in 1990 and 2017. (A) Incidence rate in 1990; (B) death rate in 1990; (C) DALY rate in 1990; (D) incidence rate in 2017; (E) death rate in 2017; (F) DALY rate in 2017. 


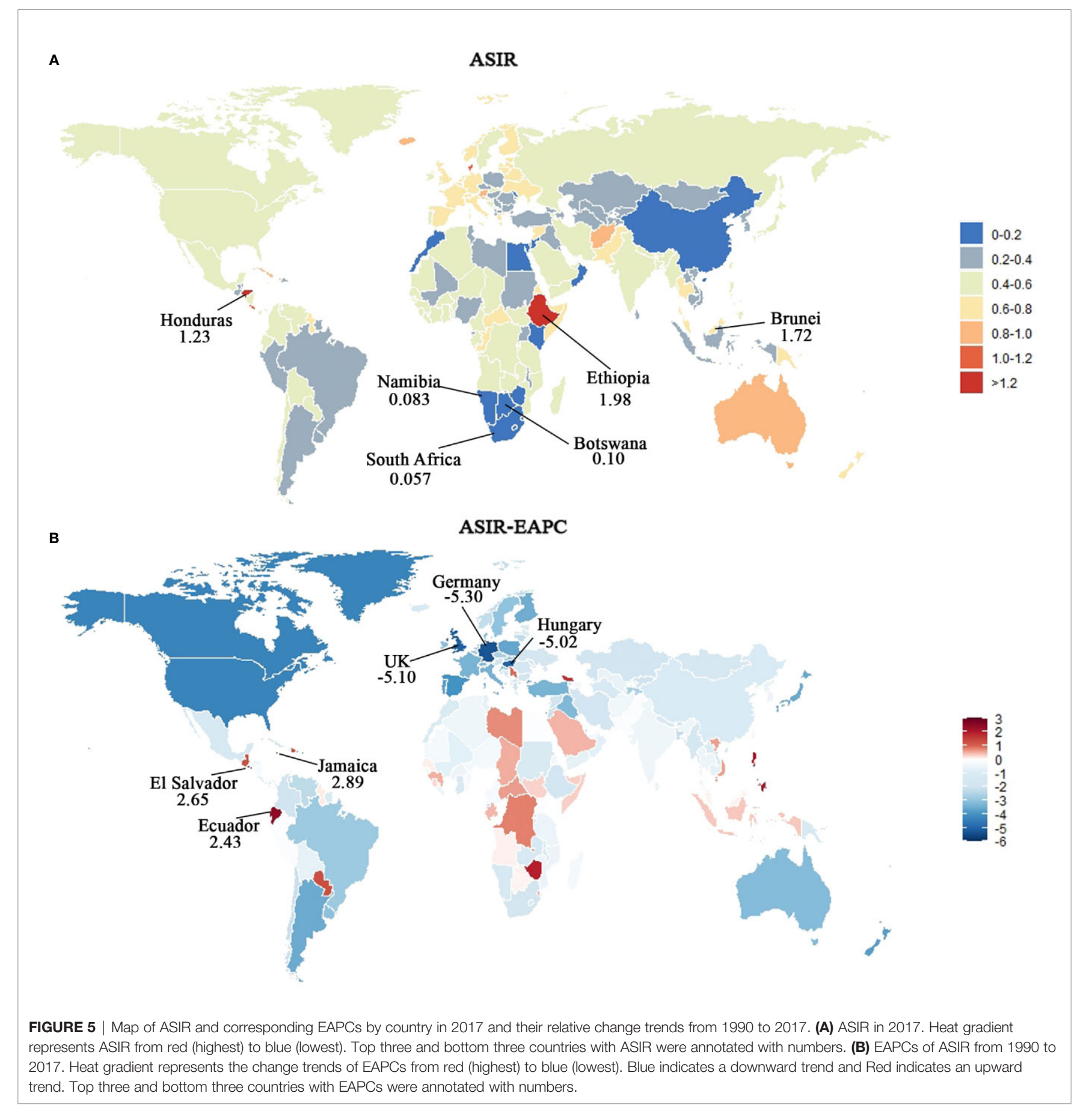

of incidence, deaths, and DALYs were stable between 1990 and 2017 globally. On the contrary, the ASIR, ASDR, and agestandardized DALY rates of CML showed decreasing trends. Especially since 1997, the year of approval of the first TKI, the decline of ASRs became more pronounced, pointing to the contribution of TKI treatment to the changes in the global patterns of CML. In addition, the burden of CML varied widely across different SDI-based regions. Our analysis showed that the age-adjusted incidence, mortality, and DALY rates of CML in the high- and high-middle-SDI regions decreased steadily over the past 28 years. However, in the low-SDI regions, these rates did not improve significantly, and they had the highest incidence, and mortality. We also revealed that the lower the SDI, the higher was the proportion of incidence and deaths in the younger age groups.

Previous studies have demonstrated that cancer survival rates between countries with different income levels differ for reasons such as variations in effective treatment, specialized 
TABLE 2 | Death cases and ASDR in 1990 and 2017 and its change trends from 1990 to 2017.

\begin{tabular}{|c|c|c|c|c|c|}
\hline & \multicolumn{2}{|c|}{1990} & \multicolumn{2}{|c|}{2017} & \multirow{2}{*}{$\begin{array}{c}1990-2017 \\
\text { EAPC } \\
\text { No. }(95 \% \mathrm{Cl})\end{array}$} \\
\hline & $\begin{array}{c}\text { Death cases } \\
\text { No. }{ }^{*} 10^{2}(95 \% \text { UI) }\end{array}$ & $\begin{array}{c}\text { ASDR } \\
\text { Per } 100,000 \\
\text { No. }(95 \% \text { UI) }\end{array}$ & $\begin{array}{c}\text { Death cases } \\
\text { No. }{ }^{*} 10^{2}(95 \% \text { UI) }\end{array}$ & $\begin{array}{c}\text { ASDR } \\
\text { Per } 100,000 \\
\text { No. }(95 \% \text { UI) }\end{array}$ & \\
\hline Overall & 734.92(666.26-806.31) & 15.96(14.6-17.44) & 240.54(222.33-260.72) & $0.31(0.28-0.33)$ & $-2.74(-9.31-4.31)$ \\
\hline \multicolumn{6}{|l|}{ Sex } \\
\hline Female & 351.99(299.2-414.08) & 14.78(17.36-12.72) & 111.39(96.83-127.32) & $0.26(0.23-0.3)$ & $-2.88(-9.9-4.69)$ \\
\hline Male & 382.94(349.15-415.35) & 17.48(18.92-16.08) & 129.15(116.44-140.91) & $0.36(0.33-0.39)$ & $-2.63(-8.7-3.84)$ \\
\hline \multicolumn{6}{|l|}{ Socio-demographic index } \\
\hline High SDI & 286.21(278.31-294.78) & 24.23(24.96-23.53) & 70.66(67.96-73.85) & $0.31(0.3-0.32)$ & $-4.51(-10.31-1.65)$ \\
\hline High-middle SDI & 129.2(112.36-144.95) & $12.17(13.57-10.67)$ & 33.92(31.8-37.07) & $0.2(0.18-0.21)$ & $-2.94(-10.73-5.54)$ \\
\hline Middle SDI & 110.8(96.98-129.21) & 8.68(10.09-7.68) & 45.79(39.63-49.07) & $0.21(0.18-0.23)$ & $-1.28(-9.92-8.2)$ \\
\hline Low-middle SDI & 100.72(86-128.82) & 13.25(16.8-11.49) & $45.12(39.06-52.46)$ & $0.37(0.32-0.43)$ & $-0.7(-7.51-6.6)$ \\
\hline Low SDI & $106.41(82.75-137.73)$ & 23.14(29.19-18.45) & 44.67(37.71-50.65) & $0.6(0.5-0.68)$ & $-1.03(-6.37-4.63)$ \\
\hline \multicolumn{6}{|l|}{ Region } \\
\hline Andean Latin America & $2.58(2.16-2.98)$ & $9.01(10.28-7.53)$ & 1.46(1.19-1.64) & $0.26(0.21-0.29)$ & $-0.28(-8.51-8.68)$ \\
\hline Australasia & 6.8(6.49-7.13) & 29.19(30.62-27.86) & $2.13(1.89-2.43)$ & $0.44(0.39-0.5)$ & $-4.52(-9.53-0.77)$ \\
\hline Caribbean & $6.72(6.12-7.52)$ & 22.56(25.15-20.54) & $2.45(2.21-2.73)$ & $0.48(0.44-0.54)$ & $-2.08(-7.7-3.88)$ \\
\hline Central Asia & 7.39(6.31-8.47) & 12.76(14.6-10.93) & $1.98(1.75-2.2)$ & $0.25(0.22-0.27)$ & $-1.62(-9.21-6.61)$ \\
\hline Central Europe & 27.55(26.09-29.01) & 19.12(20.16-18.14) & 6.42(6.06-6.83) & $0.31(0.29-0.33)$ & $-2.98(-9.17-3.64)$ \\
\hline Central Latin America & 19.46(18.71-20.35) & 16.88(17.64-16.22) & 8.55(8.06-9.03) & $0.36(0.34-0.38)$ & $-1.97(-8.25-4.74)$ \\
\hline Central Sub-Saharan Africa & 4.86(3.14-6.32) & 14.47(18.41-9.83) & 3.02(2.21-3.75) & $0.54(0.39-0.67)$ & $0.55(-5.65-7.15)$ \\
\hline East Asia & 62.56(46.66-79.09) & $5.02(6.27-3.85)$ & 11.79(9.94-13.91) & $0.06(0.05-0.07)$ & $-3.49(-16.86-12.04)$ \\
\hline Eastern Europe & 44.34(40.1-50.69) & 16.35(18.62-14.87) & $12.37(11.71-13.09)$ & $0.38(0.36-0.4)$ & $-1.52(-7.72-5.1)$ \\
\hline Eastern Sub-Saharan Africa & $37.33(26.7-51.97)$ & $32.79(43.98-24.4)$ & 13.98(10.71-16.95) & $0.77(0.59-0.94)$ & $-1.4(-5.98-3.41)$ \\
\hline High-income Asia Pacific & 34.19(32.85-35.83) & 17.16(18.02-16.47) & $6.01(5.64-6.4)$ & $0.14(0.13-0.15)$ & $-5.14(-13.28-3.76)$ \\
\hline High-income North America & 73.89(71.98-76.1) & 22.53(23.2-21.93) & 15.6(14.97-16.43) & $0.26(0.25-0.27)$ & $-5.15(-11.56-1.71)$ \\
\hline North Africa and Middle East & $33.74(24.28-41.55)$ & $14.58(17.84-10.63)$ & 12.32(10.13-14.35) & $0.28(0.23-0.32)$ & $-2.25(-9.22-5.25)$ \\
\hline Oceania & 1.06(0.79-1.44) & 23.03(30.17-17.52) & $0.41(0.31-0.56)$ & $0.47(0.37-0.62)$ & $-1.28(-7.18-4.99)$ \\
\hline South Asia & $140.17(116.31-172.87)$ & 17.35(21.55-14.7) & 66.6(57.12-76.5) & $0.49(0.43-0.57)$ & $-0.8(-6.73-5.51)$ \\
\hline Southeast Asia & $36.28(28.98-52.09)$ & $10.3(15-8.45)$ & 16.51(13.01-19.91) & $0.28(0.22-0.33)$ & $-0.73(-8.48-7.69)$ \\
\hline Southern Latin America & $9.22(8.5-9.93)$ & 19.28(20.78-17.79) & 2.29(2.05-2.52) & $0.28(0.25-0.31)$ & $-3.66(-9.9-3.02)$ \\
\hline Southern Sub-Saharan Africa & 0.98(0.73-1.19) & 2.63(3.24-1.96) & $0.37(0.28-0.46)$ & $0.06(0.05-0.08)$ & $-0.79(-15.48-16.45)$ \\
\hline Tropical Latin America & 20.5(19.63-21.45) & $16.99(17.74-16.31)$ & 5.97(5.73-6.29) & $0.26(0.25-0.28)$ & $-3.06(-9.75-4.14)$ \\
\hline Western Europe & $150.45(144.73-156.46)$ & 28.93(30.07-27.85) & $42.45(40.25-44.87)$ & $0.44(0.41-0.46)$ & $-4.03(-9.01-1.22)$ \\
\hline Western Sub-Saharan Africa & 14.87(11.7-18.64) & 12.23(15.37-9.58) & 7.87(6.28-9.9) & $0.37(0.3-0.47)$ & $-0.21(-7.3-7.43)$ \\
\hline
\end{tabular}

$A S D R$, age-standardized death rate; EAPC, estimated annual percentage change.

health care, insurance status, and cancer prevention programs $(5,16,17)$. We speculate that out of these factors, the most important explanation of our findings might be the lack of basic diagnostic and treatment options in the low-income countries that were well-established in the high-income countries, including the non-availability of TKI therapy or non-adherence to that (6). Besides, due to the lack of widelyavailable monitoring technology in the low-SDI regions, the efficacy of TKI treatment couldn't be evaluated in time, which might be another important factor. These findings raise an alarm that rational allocation of existing resources is urgently needed to reduce the burden of CML in the lowSDI regions.

Consistent with previous findings, the incidence and mortality of CML varied across GBD regions and countries (68, 18-21). ASIR showed an upward trend in three GBD regions: Central Sub-Saharan Africa, Andean Latin America, and Southeast Asia. Moreover, Central Sub-Saharan Africa also showed an upward trend of ASDR, and age-standardized DALY, suggesting the highest burden of CML in this region.
The geographic variations in the incidence of CML could partly be attributed to the methodological factors (6). In particular, the inclusion of referral patients in some registries might have resulted in an overestimation of the incidence of CML, whereas incomplete reporting of new CML cases might lead to a low incidence. Although these methodological issues cannot be ruled out as the cause of the divergence in CML incidence, the possibility of a real variation between different regions cannot be excluded. In fact, geographical differences of CML incidence have been shown in few previous studies. A report from the EUTOS registry (22), based on epidemiological data from 20 European countries between 2008 and 2012, showed that the CML incidence varied from 0.76 (UK) to 1.96 (Finland) per 100,000 persons. Another report from Chen Y. et al. (7, 23) suggested that the incidence of CML is lower in some Asian countries, which was consistent with our findings. Interestingly, similar to the regional patterns of Hodgkin's Lymphoma (24), the countries with the highest incidence and mortality of CML were mostly coastal or island countries, such as Ethiopia, Brunei, Honduras, Seychelles, Denmark, and Costa Rica. The causes of 
A

ASDR

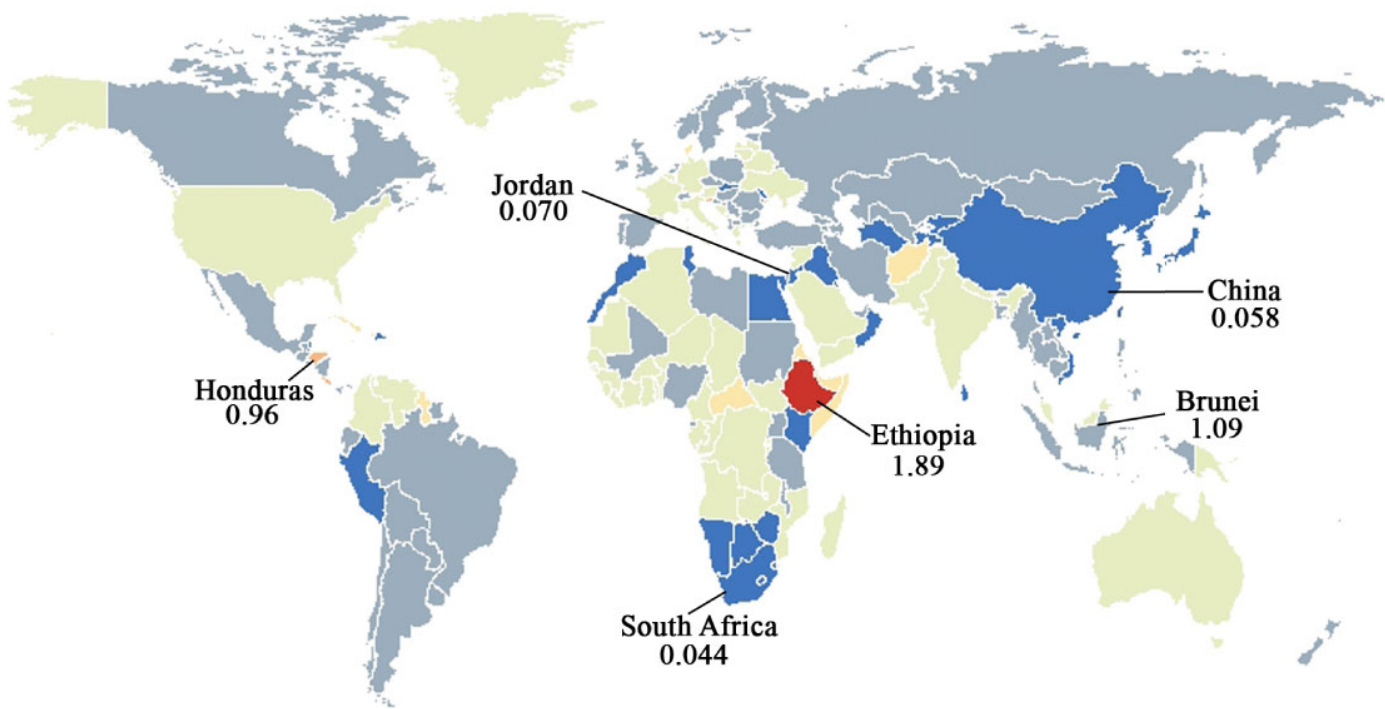

B

ASDR-EAPC
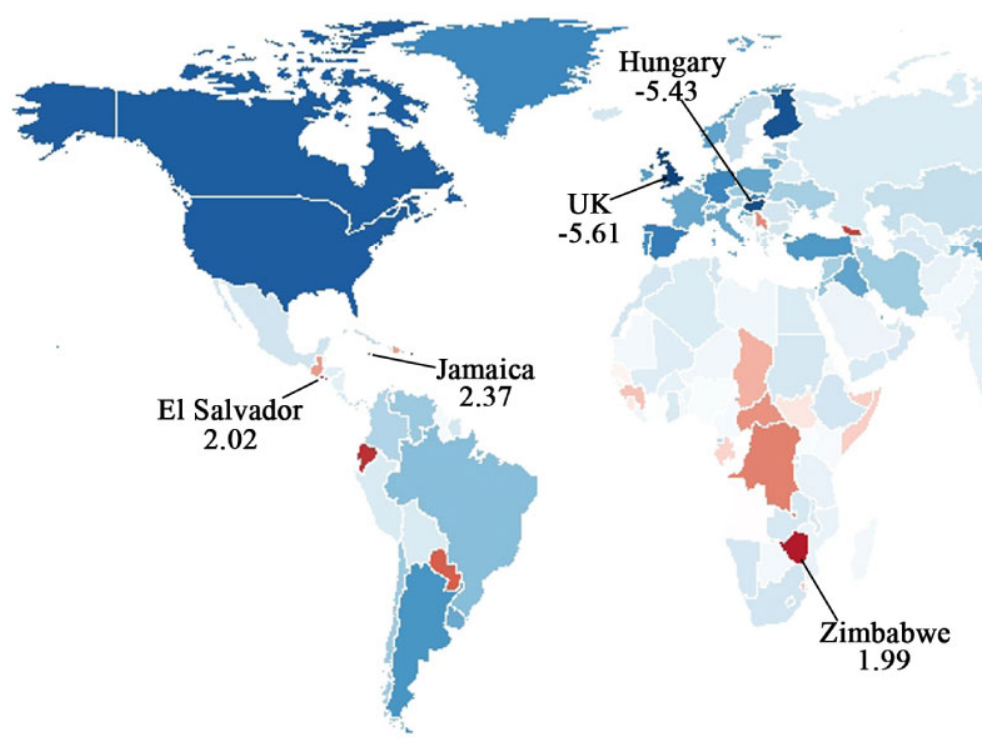

FIGURE 6 | Map of ASDR and corresponding EAPCs by country in 2017 and their relative change trends from 1990 to 2017. (A) ASDR in 2017. Heat gradient represents ASIR from red (highest) to blue (lowest). Top three and bottom three countries with ASDR were annotated with numbers. (B) EAPCs of ASDR from 1990 to 2017. Heat gradient represents the change trends of EAPCs from red (highest) to blue (lowest). Blue indicates a downward trend and red indicates an upward trend. Top three and bottom three countries with EAPCs were annotated with numbers.

this phenomenon remain to be elucidated and require further study.

CML is a clonal myeloproliferative neoplasm characterized by a reciprocal translocation between chromosomes 9 and 22 . Although the pathogenesis is well known, the etiology of CML remains incompletely understood. A recent study based on the
Swedish Cancer Registry showed no evidence that CML was heritable (25). To date, ionizing radiation is the only confirmed risk factor for CML (26). The association between smoking or benzene exposure and CML has also been reported in few studies, but this needs to be confirmed (2629). The analysis of epidemiological characteristics of CML 
TABLE 3 | DALY and age-standardized DALY rate in 1990 and 2017 and its change trends from 1990 to 2017.

\begin{tabular}{|c|c|c|c|c|c|}
\hline & \multicolumn{2}{|c|}{1990} & \multicolumn{2}{|c|}{2017} & \multirow{2}{*}{$\begin{array}{c}\text { 1990-2017 } \\
\text { EAPC } \\
\text { No. }(95 \% \mathrm{Cl})\end{array}$} \\
\hline & $\begin{array}{c}\text { DALY } \\
\text { No. }{ }^{*} 10^{3}(95 \% \text { UI) }\end{array}$ & $\begin{array}{l}\text { Age-standardized } \\
\text { DALY rate } \\
\text { Per } 100,000 \\
\text { No. }(95 \% \text { UI) }\end{array}$ & $\begin{array}{c}\text { DALY } \\
\text { No. }{ }^{*} 10^{3}(95 \% \text { UI) }\end{array}$ & $\begin{array}{l}\text { Age-standardized } \\
\text { DALY rate } \\
\text { Per } 100,000 \\
\text { No. }(95 \% \text { UI) }\end{array}$ & \\
\hline Overall & 317.52 (295.9-340.66) & $0.75(0.71-0.8)$ & 654.98(594.73-712.95) & $8.17(7.42-8.88)$ & $-2.82(-4.12-1.49)$ \\
\hline \multicolumn{6}{|l|}{ Sex } \\
\hline Female & $151.96(135.82-171.91)$ & $0.67(0.61-0.75)$ & $307.74(258.51-357.55)$ & $7.48(6.27-8.68)$ & $-2.88(-4.24-1.49)$ \\
\hline Male & 165.57 (153.46-177.23) & $0.86(0.8-0.92)$ & $347.24(308.67-383.12)$ & 9(8.01-9.9) & $-2.77(-4.01-1.51)$ \\
\hline \multicolumn{6}{|l|}{ Socio-demographic index } \\
\hline High SDI & $163.42(157.15-169.51)$ & $1.34(1.29-1.38)$ & $125.91(121.16-131.32)$ & $6.81(6.57-7.08)$ & $-5.25(-6.46-4.02)$ \\
\hline High-middle SDI & $49.58(43.76-55.26)$ & $0.49(0.43-0.54)$ & 91.51(85-100.26) & 5.26(4.9-5.76) & $-3.47(-4.97-1.94)$ \\
\hline Middle SDI & $38.31(34.17-44.49)$ & $0.33(0.3-0.39)$ & $137.8(120.1-148.24)$ & $6.03(5.25-6.47)$ & $-1.58(-3.23-0.09)$ \\
\hline Low-middle SDI & $32.8(28.42-41.59)$ & $0.48(0.42-0.61)$ & 146.73(127.78-171.91) & 10.33(8.95-12.07) & $-0.99(-2.28-0.32)$ \\
\hline Low SDI & $32.8(25.9-41.69)$ & $0.81(0.67-1.01)$ & $151.91(126.85-172.16)$ & $16.71(14.03-18.84)$ & $-1.34(-2.35-0.32)$ \\
\hline \multicolumn{6}{|l|}{ Region } \\
\hline Andean Latin America & $0.87(0.73-1)$ & $0.34(0.28-0.38)$ & 4.49(3.61-5.14) & $7.68(6.21-8.76)$ & $-0.45(-2-1.13)$ \\
\hline Australasia & $3.87(3.36-4.44)$ & $1.64(1.43-1.87)$ & 4.02(3.54-4.58) & 9.87(8.64-11.2) & $-4.72(-5.78-3.65)$ \\
\hline Caribbean & $2.64(2.41-2.91)$ & $0.93(0.85-1.02)$ & 6.66(5.9-7.62) & $13.28(11.77-15.18)$ & $-2.16(-3.26-1.05)$ \\
\hline Central Asia & $2.52(2.16-2.88)$ & $0.45(0.39-0.51)$ & 6.69(5.93-7.44) & 7.62(6.77-8.45) & $-2(-3.37-0.62)$ \\
\hline Central Europe & $11.11(10.48-11.77)$ & $0.77(0.73-0.82)$ & 13.52(12.8-14.32) & 7.53(7.14-7.98) & $-3.65(-4.89-2.4)$ \\
\hline Central Latin America & $6.98(6.69-7.4)$ & $0.66(0.64-0.7)$ & 24.79(23.35-26.32) & 9.98(9.41-10.58) & $-2.28(-3.49-1.05)$ \\
\hline Central Sub-Saharan Africa & $1.49(0.99-1.92)$ & $0.51(0.35-0.64)$ & 11.01(7.99-13.7) & 14.97(10.91-18.55) & $0.26(-0.92-1.45)$ \\
\hline East Asia & $22.64(17.43-28.02)$ & $0.19(0.16-0.24)$ & $36.19(30.76-43.35)$ & $1.9(1.63-2.27)$ & $-4.29(-6.71-1.8)$ \\
\hline Eastern Europe & $18.01(15.98-20.72)$ & $0.66(0.59-0.75)$ & $32.48(30.66-34.41)$ & $10.85(10.26-11.55)$ & $-1.94(-3.1-0.77)$ \\
\hline Eastern Sub-Saharan Africa & 11.1 (8.15-15.09) & $1.11(0.86-1.49)$ & 51.15(39.01-62.33) & 21.72(16.64-26.33) & $-1.81(-2.67-0.95)$ \\
\hline High-income Asia Pacific & $17.08(15.68-18.57)$ & $0.87(0.79-0.94)$ & 11.61(10.84-12.33) & 3.85(3.59-4.12) & $-5.94(-7.47-4.37)$ \\
\hline High-income North America & $34.1(32.98-35.37)$ & $1.02(0.98-1.06)$ & 31.36(29.89-33.21) & $6.14(5.82-6.5)$ & $-5.87(-7.17-4.55)$ \\
\hline North Africa and Middle East & $11.85(8.69-14.29)$ & $0.57(0.42-0.69)$ & 39.69(32.3-47.11) & $7.66(6.27-9)$ & $-2.47(-3.82-1.09)$ \\
\hline Oceania & $0.33(0.25-0.44)$ & $0.78(0.6-1)$ & $1.65(1.2-2.34)$ & 16.36(12.26-22.41) & $-1.3(-2.33-0.27)$ \\
\hline South Asia & $44.97(38.05-55.49)$ & $0.64(0.55-0.79)$ & 207.79(180.43-239.39) & 13.25(11.44-15.19) & $-1.06(-2.21-0.09)$ \\
\hline Southeast Asia & $12.41(10.18-17.69)$ & $0.39(0.33-0.57)$ & $52.2(41.39-62.2)$ & 7.87(6.24-9.39) & $-0.99(-2.46-0.5)$ \\
\hline Southern Latin America & $3.77(3.47-4.09)$ & $0.8(0.73-0.87)$ & $5.36(4.81-5.91)$ & $7(6.27-7.73)$ & $-4.08(-5.33-2.82)$ \\
\hline Southern Sub-Saharan Africa & $0.33(0.25-0.42)$ & $0.1(0.07-0.12)$ & 1.26(0.93-1.57) & 1.88(1.39-2.3) & $-1.03(-3.82-1.84)$ \\
\hline Tropical Latin America & $7.2(6.88-7.55)$ & $0.65(0.62-0.68)$ & $15.79(15.04-16.59)$ & 6.64(6.33-6.97) & $-3.68(-4.98-2.37)$ \\
\hline Western Europe & $99.5(94.04-104.66)$ & $1.78(1.69-1.87)$ & 68.46(64.8-72.73) & 8.84(8.39-9.38) & $-4.8(-5.87-3.72)$ \\
\hline Western Sub-Saharan Africa & $4.74(3.75-5.92)$ & $0.43(0.34-0.54)$ & 28.79(23.26-37.07) & 10.82(8.63-13.73) & $-0.51(-1.84-0.84)$ \\
\hline
\end{tabular}

$D A L Y$, disability-adjusted life year; EAPC, estimated annual percentage change.

may be helpful for the exploration of its etiology. Incidence of CML is rare in children and increases with age, at least up to 75-80 years, suggesting that the aging may be a risk factor of CML. The ratio of male to female incidence showed a peak in 75-80 years age group and dropped sharply after 80 years old. These results demonstrated that the occurrence of CML was related to sex, and female-related hormones might be a protective factor of CML. Moreover, the countries with the highest incidence of CML were mostly coastal or island countries. In particular, the incidence of Ethiopia was significantly higher than other countries. In-depth investigation of the environment in these countries may reveal additional clues to the causes of CML.

This study inevitably suffers from some limitations. The accuracy of the results depended on the quality of GBD data. However, in some underdeveloped regions, because of limited specialized medical care and no reliable mortality information systems, the accuracy and integrity of the data cannot be guaranteed. Besides, it can't be ignored that misdiagnosed patients might have a potential impact on the incident cases. Especially, in the 1990's, because of the poor diagnostic techniques of molecular biology, the incidence rate of CML may be underestimated. However, the misdiagnosis didn't affect the decline trend of CML incidence rate. What's more, the GBD study is based on countries and regions; therefore, the influence of race could not be analyzed.

\section{CONCLUSION}

In conclusion, based on the analysis of GHDx data, the overall incidence rates, deaths, and DALYs related to CML were stable between 1990 and 2017; however, the ASRs of CML showed a downward trend worldwide. Most of the CML burden was observed in males, especially among the older population. The low-SDI quintile had the heaviest burden of CML, especially in the regions of Andean Latin America, Central Sub-Saharan Africa, and Southeast Asia. Our analysis of the distribution, burden and trends of CML in different regions and countries 
A

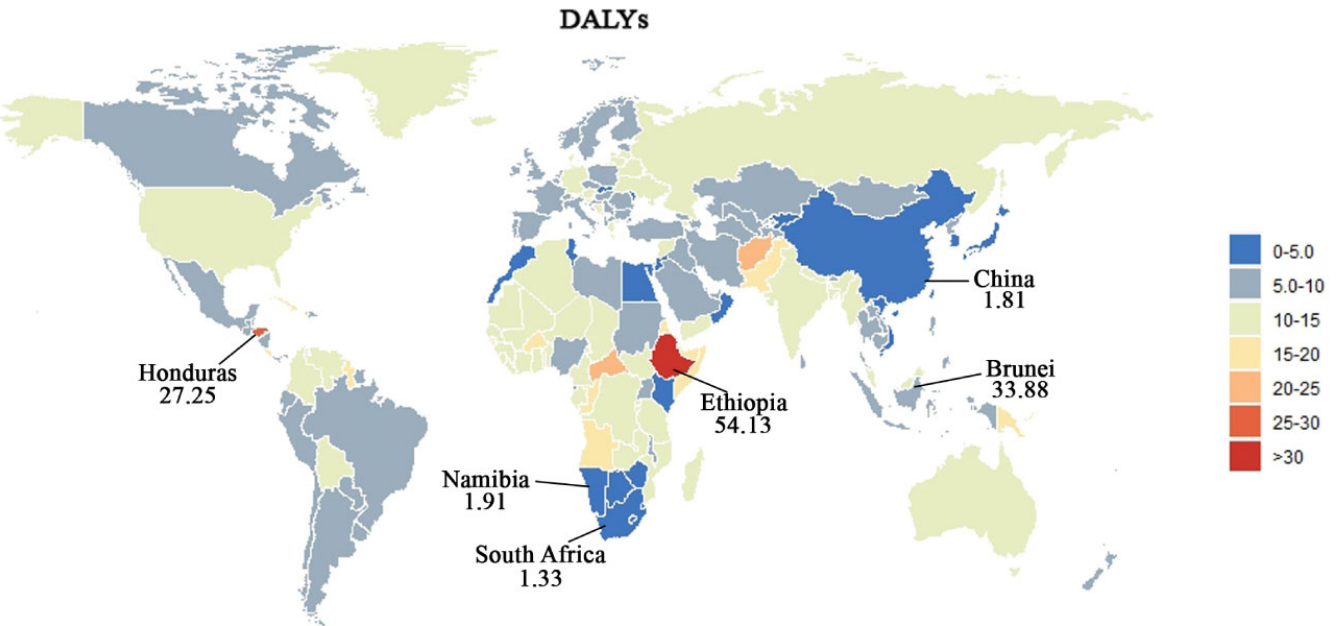

B

DALYs-EAPC

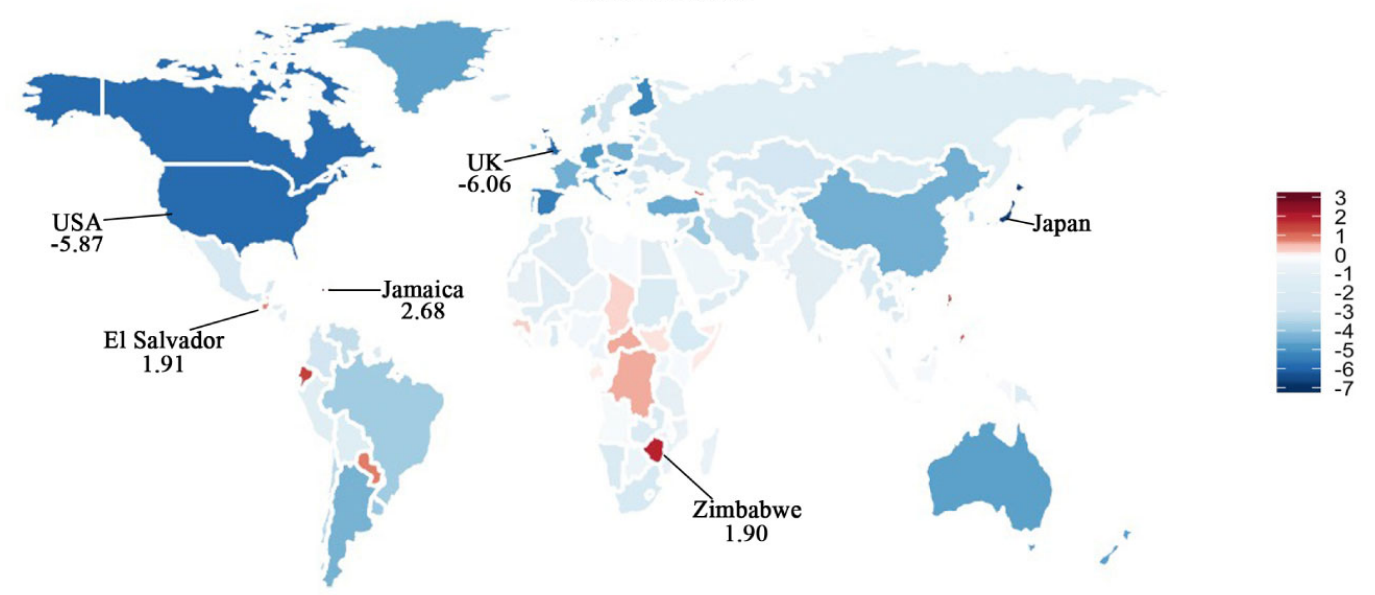

FIGURE 7 | Map of age-standardized DALY rate and corresponding EAPCs by country in 2017 and their relative change trends from 1990 to 2017. (A) Agestandardized DALY rate in 2017. Heat gradient represents age-standardized DALY rate from red (highest) to blue (lowest). Top three and bottom three countries with age-standardized DALY rate were annotated with numbers. (B) EAPCs of age-standardized DALY rate from 1990 to 2017. Heat gradient represents the change trends of EAPCs from red (highest) to blue (lowest). Blue indicates a downward trend and Red indicates an upward trend. Top three and bottom three countries with EAPCs were annotated with numbers.

may provide a direction to allocate limited resources and formulate more rational policies.

\section{DATA AVAILABILITY STATEMENT}

The original contributions presented in the study are included in the article/Supplementary Materials. Further inquiries can be directed to the corresponding authors.

\section{AUTHOR CONTRIBUTIONS}

JJ, HZ, and LY designed the study. LY and QL collected and analyzed data. LY wrote the main manuscript. QL, LY, LS, LM, $\mathrm{QH}$, and $\mathrm{LZ}$ performed the figures and tables. JJ and $\mathrm{HZ}$ reviewed the manuscript. All authors contributed to the article and approved the submitted version.

\section{FUNDING}

The research was supported by National Natural Science Foundation of China (No. 81873451).

\section{SUPPLEMENTARY MATERIAL}

The Supplementary Material for this article can be found online at: https://www.frontiersin.org/articles/10.3389/fonc. 2020. 580759/full\#supplementary-material 


\section{REFERENCES}

1. Miranda-Filho A, Piñeros M, Ferlay J, Soerjomataram I, Monnereau A, Bray F. Epidemiological patterns of leukaemia in 184 countries: a population-based study. Lancet Haematol (2018) 5(1):e14-24. doi: 10.1016/S2352-3026(17) 30232-6

2. Apperley JF. Chronic myeloid leukaemia. Lancet (2015) 385(9976):1447-59. doi: 10.1016/S0140-6736(13)62120-0

3. Hochhaus A, Larson RA, Guilhot F, Radich JP, Branford S, Hughes TP, et al. Long-Term Outcomes of Imatinib Treatment for Chronic Myeloid Leukemia. N Engl J Med (2017) 376(10):917-27. doi: 10.1056/ NEJMoa1609324

4. Huang XL, Cortes J, Kantarjian H. Estimations of the increasing prevalence and plateau prevalence of chronic myeloid leukemia in the era of tyrosine kinase inhibitor therapy. Cancer (2012) 118(12):3123-7. doi: 10.1002/cncr.26679

5. Global Burden of Disease Cancer Collaboration. Global, Regional, and National Cancer Incidence,Mortality, Years of Life Lost, Years Lived With Disability, and Disability-Adjusted Life-years for 32 Cancer Groups, 1990 to 2015: A Systematic Analysis for the Global Burden of Disease Study. JAMA Oncol (2017) 3(4):524-48. doi: 10.1001/jamaoncol.2016.5688

6. Höglund M, Sandin F, Simonsson B. Epidemiology of chronic myeloid leukaemia: an update. Ann Hematol (2015) 94 Suppl 2:S241-247. doi: 10.1007/s00277-015-2314-2

7. Chang CS, Lee K, Yang YH, Yang YH, Lin MT, Hsu CN. Estimation of CML incidence: disagreement between national cancer registry and health claims data system in Taiwan. Leuk Res (2011) 35(5):e53-4. doi: 10.1016/ j.leukres.2010.12.034

8. Chen Y, Wang H, Kantarjian H, Cortes J. Trends in chronic myeloid leukemia incidence and survival in the United States from 1975 to 2009. Leuk Lymphoma (2013) 54(7):1411-7. doi: 10.3109/10428194.2 012.745525

9. de la Fuente J, Baruchel A, Biondi A, de Bont E, Dresse MF, Suttorp M, et al. Managing children with chronic myeloid leukaemia (CML): recommendations for the management of CML in children and young people up to the age of 18 years. Br J Haematol (2014) 167(1):33-47. doi: 10.1111/bjh.12977

10. Rohrbacher M, Hasford J. Epidemiology of chronic myeloid leukaemia (CML). Best Pract Res Clin Haematol (2019) 22(3):295-302. doi: 10.1016/ j.beha.2009.07.007

11. Berger U, Maywald O, Pfirrmann M, Lahaye T, Hochhaus A, Reiter A, et al. Gender aspects in chronic myeloid leukemia: long-term results from randomized studies. Leukemia (2005) 19(6):984-89. doi: 10.1038/ sj.leu. 2403756

12. Ebrahimi H, Amini E, Pishgar F, Moghaddam SS, Nabavizadeh B, Rostamabadi Y, et al. Global, regional and national burden of bladder cancer, 1990 to 2016: results from the GBD study 2016. J Urol (2019) 201 (5):893-901. doi: 10.1097/JU.0000000000000025

13. Deng Y, Wang M, Zhou L, Zheng Y, Li N, Tian T, et al. Global burden of larynx cancer, 1990-2017: estimates from the global burden of disease 2017 study. Aging (Albany NY) (2020) 12(3):2545-83. doi: 10.18632/aging.102762

14. Naghavi M, Abajobir AA, Abbafati C, Abbas KM, Abd-Allah F, Abera SF, et al. Global, regional, and national age-sex specific mortality for 264 causes of death, 1980-2016: a systematic analysis for the Global Burden of Disease study 2016. Lancet (2017) 390(10100):1151-210. doi: 10.1016/S0140-6736(17)32152-9

15. Liu Z, Jiang Y, Yuan H, Fang Q, Cai N, Suo C, et al. The trends in incidence of primary liver cancer caused by specific etiologies: results from the Global Burden of Disease study 2016 and implications for liver cancer prevention. J Hepatol (2019) 70(4):674-83. doi: 10.1016/j.jhep.2018.12.001

16. Coleman MP. Cancer survival: global surveillance will stimulate health policy and improve equity. Lancet (2014) 383(9916):564-73. doi: 10.1016/S01406736(13)62225-4
17. Stringhini S, Berkman L, Dugravot A, Ferrie JE, Marmot M, Kivimaki M, et al. Socioeconomic status, structural and functional measures of social support, and mortality: The British Whitehall II Cohort Study, 1985-2009. Am J Epidemiol (2012) 175(12):1275-83. doi: 10.1093/aje/kwr461

18. Visser O, Trama A, Maynadie M, Marcos-Gragera R, Angelis RD, Mallone S, et al. Incidence, survival and prevalence ofmyeloid malignancies in Europe. Eur J Cancer (2012) 48(17):3257-66. doi: 10.1016/j.ejca.2012.05.024

19. Smith AG, Painter D, Howell DA, Evans P, Smith G, Patmore R, et al. Determinants of survival in patients with chronic myeloid leukaemia treated in the new era of oral therapy: findings from a UK populationbased patient cohort. BMJ Open (2014) 4(1):e004266. doi: 10.1136/ bmjopen-2013-004266

20. Gale RP, Cozen W, Goodman MT, Wang FF, Bernstein L. Decreased chronic lymphocytic leukemia incidence in Asians in Los Angeles County. Leuk Res (2000) 24(8):665-9. doi: 10.1016/S0145-2126(00)00038-2

21. Höglund M, Sandin F, Hellstrom K, Bjoreman M, Bjorkholm M, Brune M, et al. Tyrosine kinase inhibitor usage, treatment outcome, and prognostic scores in CML: report from the population-based Swedish CML registry. Blood (2013) 122(7):1284-92. doi: 10.1182/blood-2013-04-495598

22. Hoffmann V, Lindoerfer D, Thaler J, Lindoerfer D, Burgstaller S, Sertic D, et al. The EUTOS population-based registry: incidence and clinical characteristics of 2904 CML patients in 20 European Countries. Leukemia (2015) 29(6):1336-43. doi: 10.1038/leu.2015.73

23. Kim DW, Banavali SD, Bunworasate U, Goh YT, Ganly P, Huang he, et al. Chronic myeloid leukemia in the Asia-Pacificregion: current practice, challenges and opportunities in the targeted therapy era. Leuk Res (2010) 34 (11):1459-71. doi: 10.1016/j.leukres.2010.03.033

24. Zhou L, Deng Y, Li N, Zheng L, Tian T, Zhai Z, et al. Global, regional, and national burden of Hodgkin lymphoma from 1990 to 2017: estimates from the 2017 Global Burden of Disease study. J Hematol Oncol (2019) 12(1):107. doi: 10.1186/s13045-019-0799-1

25. Bjorkholm M, Kristinsson SY, Landgren O, Goldin LR. No familial aggregation in chronic myeloid leukemia. Blood (2013) 122(3):460-1. doi: 10.1182/blood-2013-05-501312

26. Musselman JR, Blair CK, Cerhan JR, Nguyen P, Hirsch B, Ross JA. Risk of adult acute and chronic myeloid leukemia with cigarette smoking and cessation. Cancer Epidemiol (2013) 7(4):410-6. doi: 10.1016/j.canep. 2013.03.012

27. Vlaanderen J, Lan Q, Kromhout H, et al. Occupational benzene exposure and the risk of chronic myeloid leukemia: a meta-analysis of cohort studies incorporating study quality dimensions. Am J Ind Med (2012) 55(9):77985. doi: 10.1002/ajim.22087

28. Lichtman MA. Is there an entity of chemically induced BCR-ABL-positive chronic myelogenous leukemia? Oncologist (2008) 13(6):645-54. doi: 10.1634/ theoncologist.2008-0057

29. Timonen TT, Ilvonen M. Contact with hospital, drugs, and chemicals as aetiological factors in leukaemia. Lancet (1978) 1(8060):350-2. doi: 10.1016/ S0140-6736(78)91079-6

Conflict of Interest: The authors declare that the research was conducted in the absence of any commercial or financial relationships that could be construed as a potential conflict of interest.

Copyright (c) 2020 Lin, Mao, Shao, Zhu, Han, Zhu, Jin and You. This is an openaccess article distributed under the terms of the Creative Commons Attribution License (CC BY). The use, distribution or reproduction in other forums is permitted, provided the original author(s) and the copyright owner(s) are credited and that the original publication in this journal is cited, in accordance with accepted academic practice. No use, distribution or reproduction is permitted which does not comply with these terms. 